\title{
Coordinated Multi-Point (CoMP) Adaptive Estimation and Prediction Schemes using Superimposed and Decomposed Channel Tracking
}

\section{Gencer Cili, Halim Yanikomeroglu, and F. Richard Yu}

Department of Systems and Computer Engineering,

Carleton University, ON, Canada

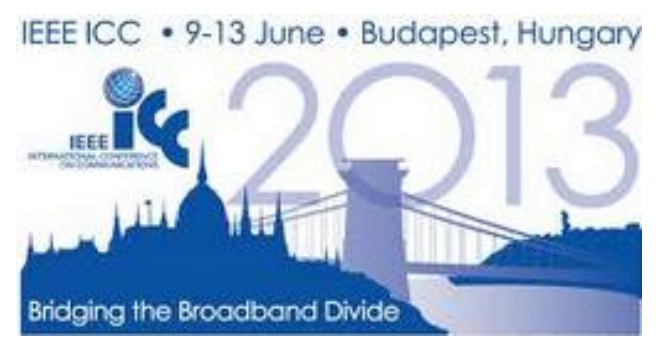




\section{Introduction}

Motivation:

- Comparison of decomposed and superimposed channel tracking methods in Coordinated Multi-Point (CoMP) networks is not studied in existing literature.

\section{Our contributions}

- Multi-point channel estimation and prediction framework to tackle the CoMP system delays and inaccurate measurements

- Comparison between superimposed versus decomposed channel estimation schemes

- CoMP adaptive switching/fallback between channel estimation schemes

- Balance the clustering accuracy versus the channel estimation computation complexity trade-off

- Effects of estimation/prediction filter length increases on CoMP performance are characterized according to users being served by various cluster sizes 


\section{CoMP Joint Transmission Procedures}

CoMP Definition: Dynamic coordination among multiple geographically separated points referred as CoMP cooperating set for downlink transmission and uplink reception

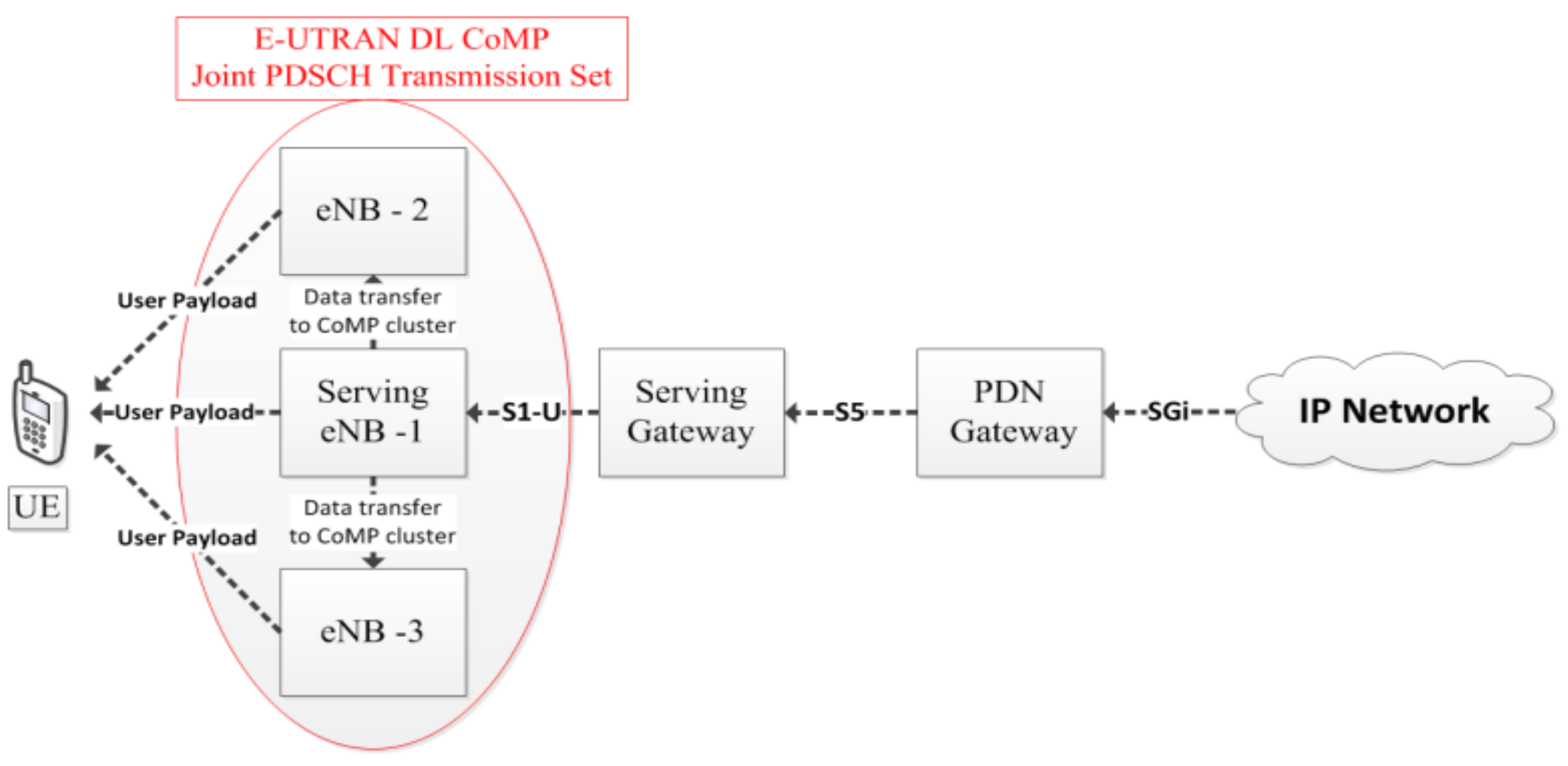




\section{CoMP Joint Transmission Procedures}

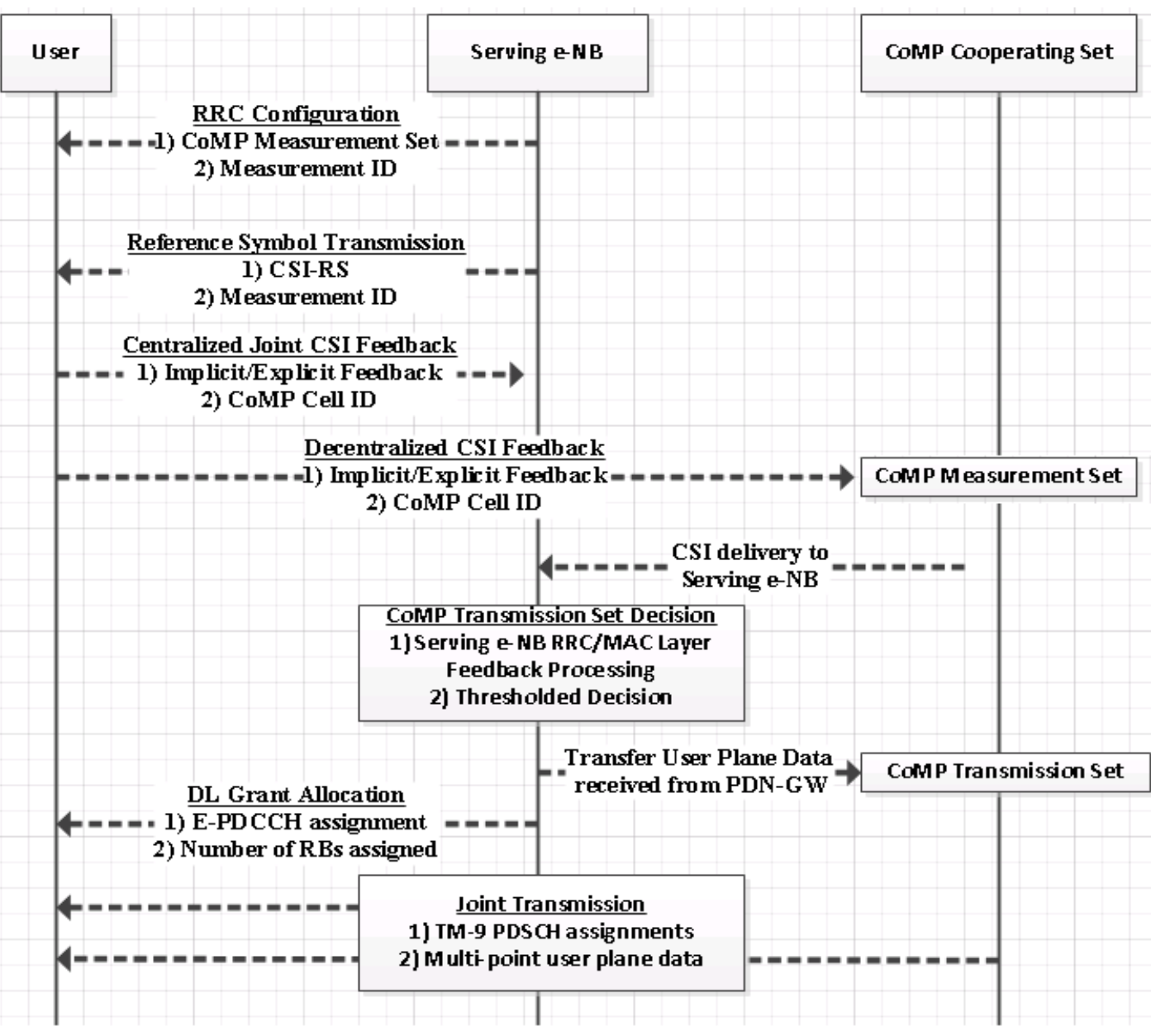

\begin{tabular}{|l|}
\hline Parameter \\
\hline $\mathbf{N}_{\text {Coop }}$ \\
\hline $\mathbf{N}_{\text {meas }}$ \\
\hline $\mathbf{N}_{\mathrm{JT}}$ \\
\hline
\end{tabular}

Assumption or Value

CoMP coordinating set

CoMP measurement set

CoMP joint transmission set

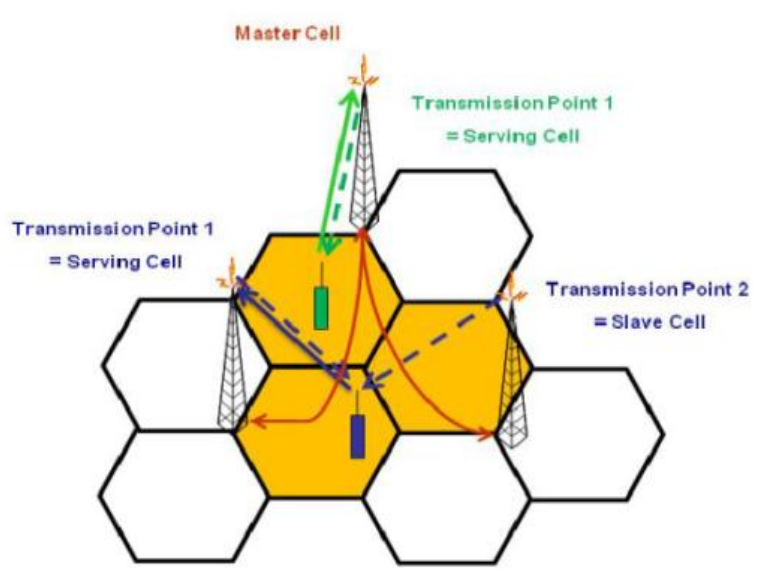




\section{Threshold Based CoMP Joint Transmission Clustering}

- Actual measured received power from eNB $n$ by user $i$ at TTI $t$ :

$$
P_{R X}(n, t, i)=P_{T X}(n)-P L(n, i)-P_{\text {Fading }}(n, i, t)
$$

- Received feedback due to estimation error + system delay:

$$
P_{R X_{-} e r r}(n, t, i)=P_{R X}(n, t-\Delta, i)+P_{e r r}(\mu, \sigma)
$$

- Threshold based Decision to Form the CoMP Transmission Set:

$$
\begin{gathered}
n_{\text {Best }}(i, t)=\underset{n}{\arg \max }\left\{P_{R X_{\text {err }}}(n, i, t)\right\} \\
\mathrm{n} \in N_{J T}(i, t) \text { if }\left|P_{R X_{\text {err }}}\left(n_{B e s t}, i, t\right)-P_{R X_{e r r}}(n, i, t)\right| \leq \nabla_{N W-J T}
\end{gathered}
$$

Note: Contents of the joint transmission set will be impacted by reported CSI feedbacks due to multi-point channel estimation errors and system delays !!! 


\section{CoMP Performance Metrics - Capacity}

- Joint PDSCH transmission (TM-9) mitigates the Inter-cell Interference

Single Point Transmission

SINR $=\frac{P_{\text {serving }}}{\sum_{i=1}^{K} P_{i}+P_{\text {Noise }}}$
CoMP Downlink Transmission

$$
S I N R_{\text {CoMP }}=\frac{P_{\text {serving }}+P_{j}+P_{m}}{\sum_{\substack{i=1 \\ i \neq j, m}}^{K} P_{i}+P_{\text {Noise }}}
$$

$\underline{\text { Total received Power from CoMP Transmission Set }}$

$$
P_{J T}(i, t)=\sum_{n \in N_{J T}} P_{R X}(n, i, t)
$$

Perceived Downlink Capacity due to CoMP

$$
C(i, t)=W(i, t) \log _{2}\left(1+\frac{P_{J T}(i, t)}{\sum_{n \in N \backslash N_{J T}(i)} P_{R X}(n, i, t)+P_{n o i s e}}\right)
$$




\section{CoMP Performance Metrics - Energy Efficiency}

\section{CoMP Power Consumption Model}

Signal Processing Power

Backhauling Power

Additional Data capacity for CoMP Backhauling Total Power Consumption of an eNB using CoMP

$$
P_{S P-C o M P}=58\left(0.87+0.1 N_{C}+0.03 N_{C}^{2}\right)
$$$$
P_{B H}=\frac{C_{B H}}{100 M b i t s / s e c} 50 \mathrm{~W}
$$

$$
C_{B H}=\frac{N_{C}\left(2 N_{C}\right) p q}{T_{S}} \text { bits } / \text { sec }
$$

\section{Power Consumption Parameters}

$N_{S}=$ Number of Sectors $N_{\frac{P A}{\text { sector }}}=$ Power amplifiers per sector $P_{T X}=$ DL Transmit Power, $C_{C}=$ Cooling Loss $C_{B B}=$ Battery Backup $N_{C}=$ Number of points in Joint Transmission $p=$ pilot density $q=$ CSI signalling $T_{S}=$ Symbol Period $P A_{e f f}=$ Power Amplifier Efficiency 


\section{CoMP Performance Metrics - Energy Efficiency}

$$
\text { Energy Efficiency }=\frac{\text { Capacity }(\text { bits } / \text { sec })}{\text { Power Consumption }(\text { Joules } / \text { sec })}=\frac{\text { bits }}{\text { Joule }}
$$

\section{Time Varying Energy Efficiency}

Joint Transmission CoMP Operation $\left(N_{C} \geq 2\right)$

Single Point Transmission $\left(N_{C}=1\right)$

$$
E E(i, t)
$$

$$
=\frac{C(i, t)}{P_{\text {CoMP }}+\left(N_{J T(i, t)}-1\right)\left(P_{\text {CoMP }}-P_{\text {Base }}\right)}
$$

\section{Notes:}

1) $P_{\text {Base }}$ has $P_{B H}=0$ since there is not need for multi-point CSI transfer to serving cell

2) $P_{S P-C o M P}=58 \mathrm{~W}$ since $N_{C}=1$ 


\section{CoMP Performance Metrics Summary}

Technical challenges of CoMP systems due to channel estimation errors and system delays:

1. Lowered Joint Transmission Cluster Degree

- Inaccurate multi-point CSI feedbacks can exclude a potential joint transmission point from the CoMP cluster unnecessarily.

- This decreases both the energy efficiency of the access network and the user perceived quality of service in terms of received downlink data rates.

\section{Expanded Joint Transmission Cluster Degree}

- Inclusion of an incorrect point in the CoMP joint transmission cluster increases the downlink data rates slightly; however, this causes significant bits/Joule energy efficiency losses since the increased power consumption of the access network is not compensated by an equal amount of downlink capacity gain for the served UEs. 


\section{Decomposed Channel Impulse Response (CIR) Estimation}

- Track each multipath delay tap of every CoMP measurement set member individually

- Perform channel estimation separately for each path

- Smoothened CIR at each path is then merged to report CSI feedback for all points

$$
\begin{gathered}
\tilde{h}_{n, i}\left(t, \tau_{l}\right)=\sum_{m=0}^{M_{U E}-1} w(m) \hat{h}\left(t-m, \tau_{l}\right) \quad w(j)>w(k) \forall j<k \\
\tilde{h}_{n, i}\left(t, \tau_{l}\right)=\left[\left(R_{h}\left(\Delta t, \tau_{l}\right)+\sigma_{n o i s e}^{2} I_{M_{U E} x M_{U E}}\right)^{-1} r_{h}\left(\Delta t, \tau_{l}\right)\right]^{H} \widehat{\boldsymbol{h}}_{t, \ldots, t-M_{U E}+1 ; \tau_{l}} \\
\widehat{\boldsymbol{h}}_{t, \ldots, t-M+1 ; \tau_{l}}=\left[\hat{h}\left(t, \tau_{l}\right) \ldots . \hat{h}\left(t-M_{U E}+1, \tau_{l}\right)\right]^{T} \\
R_{h}\left(\Delta t=M_{U E}-1, \tau_{l}\right)=E\left[h\left(t-M_{U E}+1, \tau_{l}\right) h\left(t, \tau_{l}\right)^{*}\right]
\end{gathered}
$$




\section{Superimposed Channel Impulse Response (CIR) Estimation}

- Track the superimposed time-varying CIR coefficients instead of separate CIR realizations at each path

- Yields less accurate CSI estimates compared to decomposed multipath tracking

- Multi-point channel estimation complexity of the UE is decreased significantly

- Smaller auto-correlation filter and channel estimation filter input buffers

$$
\begin{gathered}
\tilde{h}_{n, i}(t)=\left[\left(R_{h}(\Delta t)+\sigma_{n o i s e}^{2} I_{M_{U E}} x M_{U E}\right)^{-1} r_{h}(\Delta t)\right]^{H} \widehat{\boldsymbol{h}}_{t, \ldots, t-M_{U E}+1} \\
\widehat{\boldsymbol{h}}_{t, \ldots t-M+1 ; \tau_{l}}=\left[\sum_{l=1}^{L} \hat{h}\left(t, \tau_{l}\right), \ldots, \sum_{l=1}^{L} \hat{h}\left(t-M_{U E}+1, \tau_{l}\right)\right]^{T} \\
R_{h}(\Delta t)=\int_{l=1}^{L} R_{h}\left(\Delta t, \tau_{l}\right) d \tau_{l}
\end{gathered}
$$




\section{Performance Analysis of Multi-point Channel Estimation Schemes}

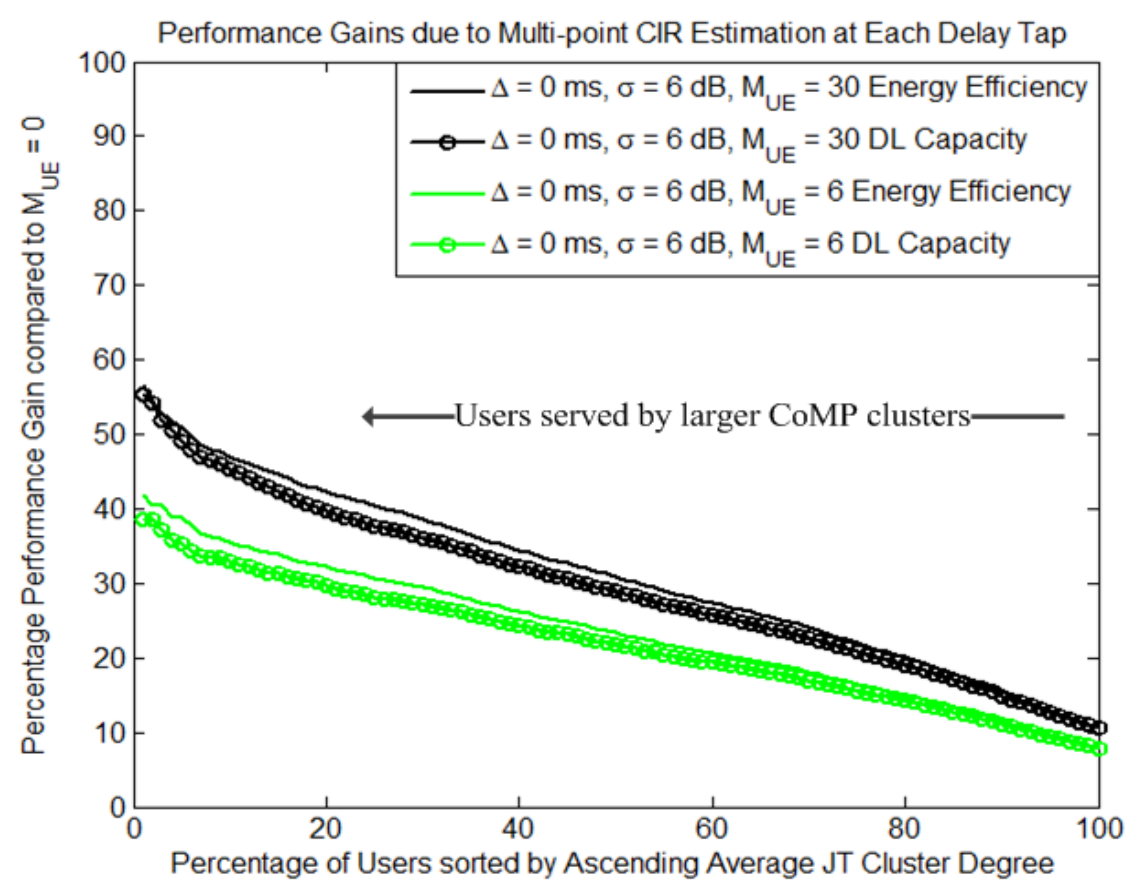

Decomposed multi-point channel estimation

- Better performance for users being served by large clusters

- Increased computational burden on the UE firmware to track each path separately

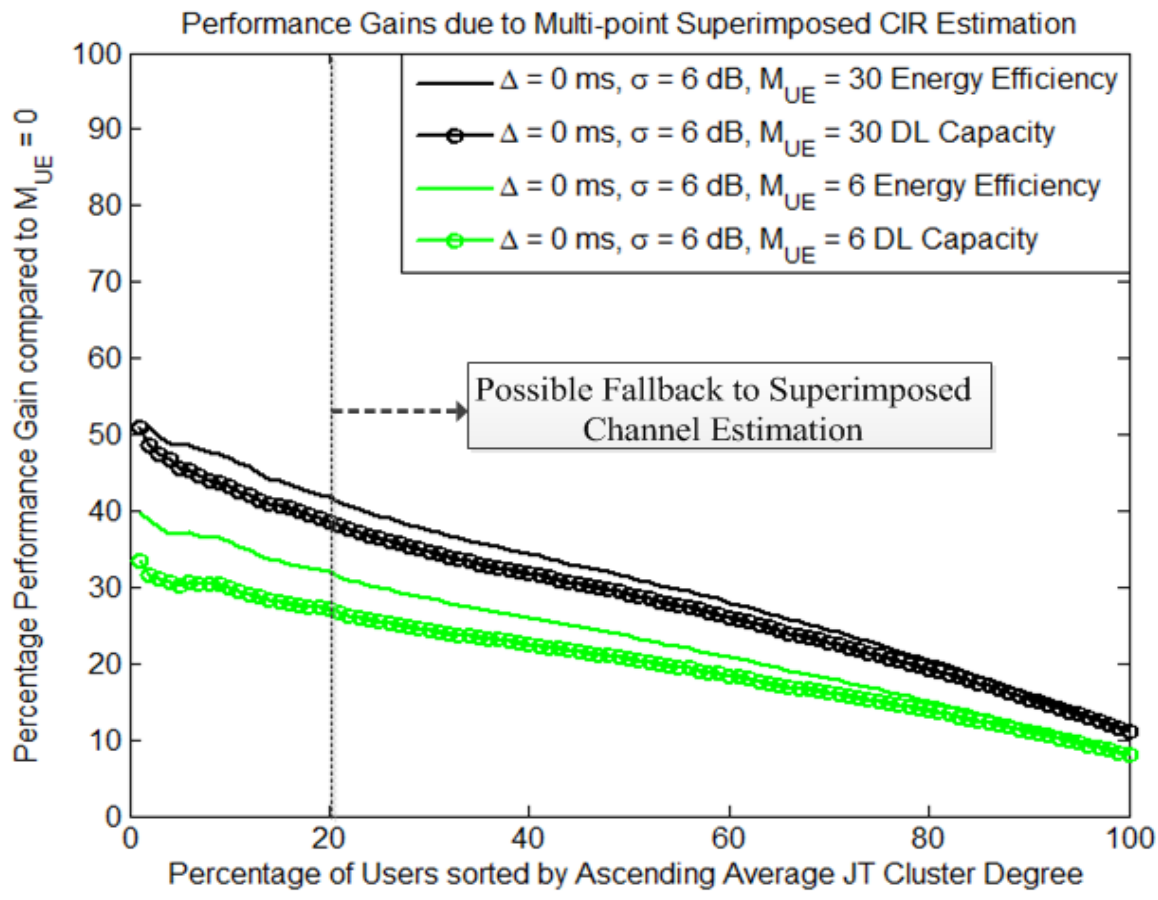

Superimposed multi-point channel estimation

- Energy efficiency and capacity degradation for users being served by large clusters

- Faster channel estimation computation time and decreased CSI feedback delay 


\section{CoMP Adaptive Channel Estimation Scheme Switching}

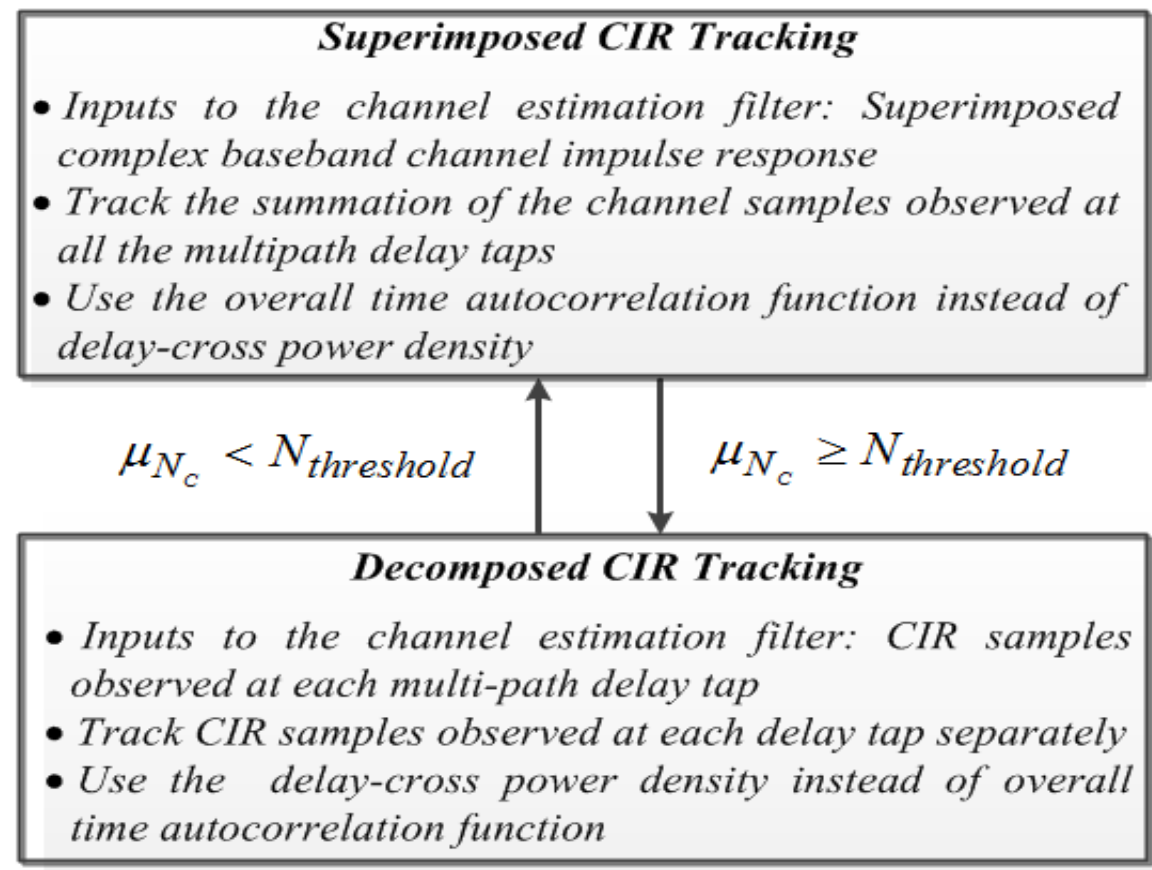

\section{CoMP cluster degree adaptive switching}

- Use Decomposed channel estimation when the wireless device is receiving downlink CoMP joint transmission from a large cluster

- Fallback to superimposed estimation if cluster size decreases
- Define critical measured received power zone as:

$\nabla_{N W-J T}-$ Threshold $_{P_{-} J T} \leq \Delta_{j, t} \leq \nabla_{N W-J T}+$ Threshold $_{P_{-} J T}$

$$
\Delta_{j, t}=P_{R X}\left(n_{\text {best }}, t\right)-P_{R X}\left(n_{j}, t\right)
$$

$\nabla_{N W-J T}=\mathrm{NW}$ configured CoMP clustering threshold

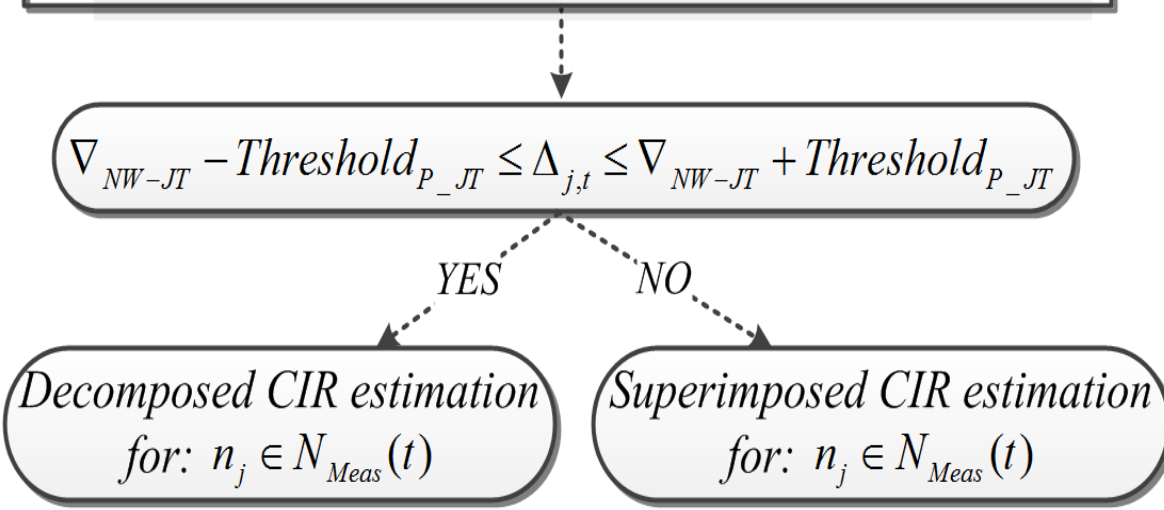

\section{CoMP received power adaptive switching}

- UEs increase the channel estimation computation complexity only for the critical points that require extremely accurate CSI feedback for joint transmission set clustering decisions 


\section{Multi-Point Channel Impulse Response (CIR) Prediction}

\begin{tabular}{|l|l|}
\hline Parameter & Representation \\
\hline$h_{n, i}$ & Predicted CIR samples by the serving e-NB \\
\hline$\tilde{h}_{n, i}$ & Estimated CIR samples by the UE \\
\hline$p \in[1, \ldots, P]$ & Prediction range in terms of number of TTIs \\
\hline$M_{N W}$ & Prediction filter length used by the serving e-NB \\
\hline
\end{tabular}

1. Serving e-NB performs the prediction at $P$ steps using:

$$
\breve{h}_{n, i}(t+p)=\sum_{\substack{m=1 \\ p>1}}^{p-1} \breve{h}_{n, i}(t+p-m) w(m)+\sum_{m=p}^{M_{N W}} \tilde{h}_{n, i}(t+p-m) w(m),
$$

2. Filter inputs, predicted CIR autocorrelation matrix and filter coefficients are updated at every step

3. Currently predicted CIR sample replaces the most outdated CIR sample for the filter input at each step $p$ 


\section{Performance improvement of CoMP systems due to multi-point channel estimation and prediction schemes}

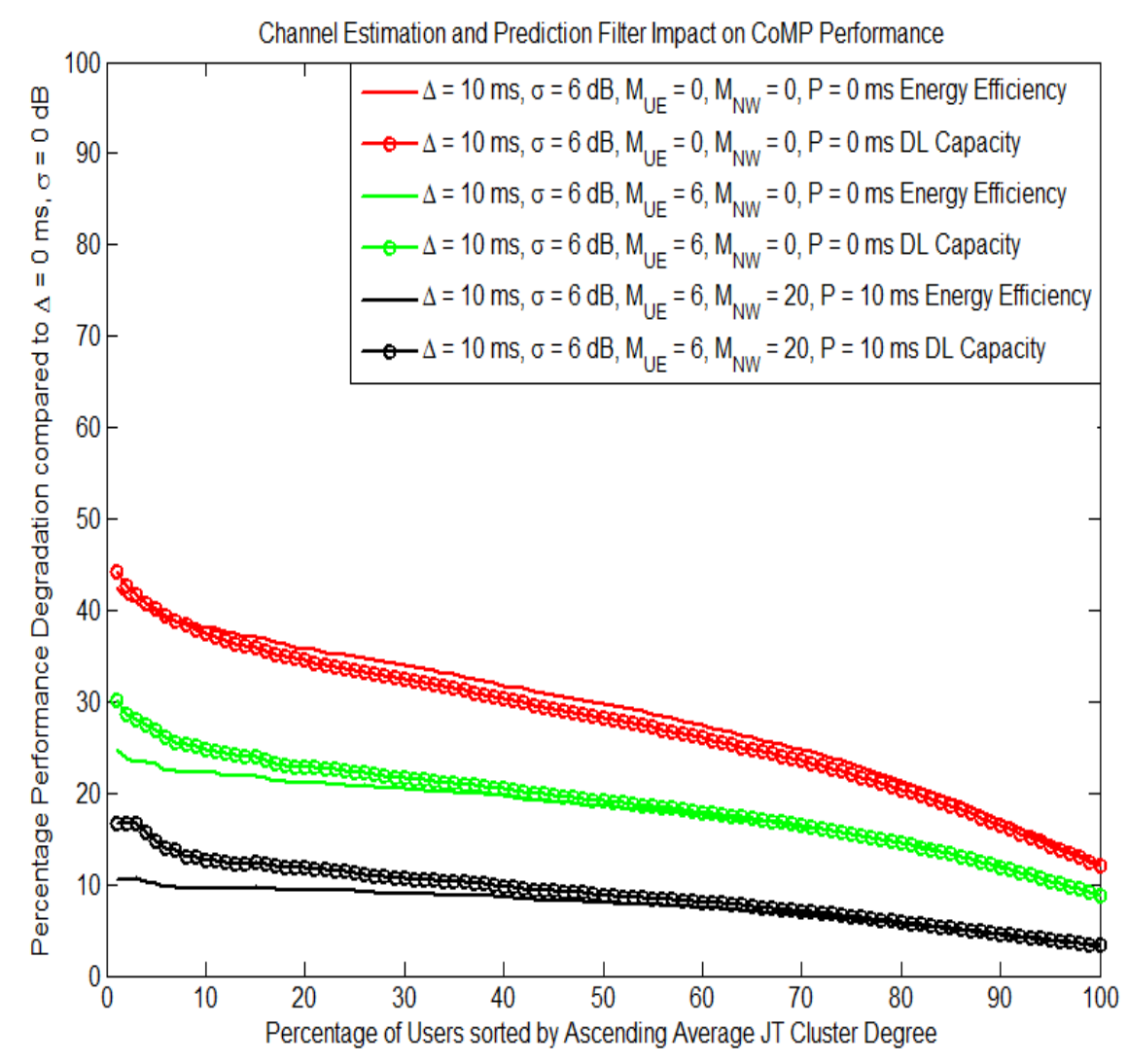

- Multi-point channel estimation procedures performed by the UEs are enough to tackle the channel estimation errors

- However, CoMP system delays still create performance degradations due to outdated CSI feedbacks

- Serving e-NB should predict how the multi-point CIRs will change at the time of the joint data transmission

- CIR prediction range $P$ is set to the system delay observed in the channel to maximize the performance gains due to prediction

- Estimation and prediction filter lengths and the prediction ranges should be adapted according to the served UE's CoMP characteristics 


\section{CONCLUSION \& SUMMARY}

- UEs should choose to switch dynamically between the two channel estimation schemes depending on the recently observed CoMP characteristics

- UEs should balance the optimal trade-off between CoMP capacity and energy efficiency performance gains versus computation complexity

- UEs that are being served by larger clusters should use decomposed CIR estimation and tracking, and fallback to superimposed tracking method when the cluster size decreases

- Use decomposed CIR estimation tracking only for the points that have received power values which are close to the joint transmission cluster threshold or for the points that are recently added to the CoMP measurement set 


\section{FUTURE WORK}

- Utilize CoMP adaptive channel estimation scheme switching method jointly with the CoMP adaptive filter length choices

- Decomposed channel estimation scheme will be further enhanced to have various filter lengths for each multipath component of every CoMP measurement set member 


\section{REFERENCES}

[1] P. Marsch and G. P. Fettweis. Coordinated Multi-Point in Mobile Communications. New York, USA: Cambridge University Press, 2011, pp. 1-460.

[2] 3GPP TS 36.819 V11.1.0, "Coordinated multi-point operation for LTE physical layer aspects," Dec. 2011.

[3] G. Cili, H. Yanikomeroglu, and F. R. Yu, "Energy efficiency and capacity evaluation of LTE-Advanced downlink CoMP schemes subject to channel estimation errors and system delay," submitted to IEEE VTC'13, Las Vegas, USA, September 2013.

[4] L. Somasegaran, "Channel Estimation and Prediction in UMTS LTE," M.S. thesis, Institute of Electronic Systems, Aalborg University, Denmark, 2007.

[5] A. Duel-Hallen, "Fading channel prediction for mobile radio adaptive transmission systems," Proc. IEEE, vol 95, no. 12, pp. 2299-2313, Dec. 2007.

[6] D. Aronsson, "Channel Estimation and Prediction for MTMO OFDM Systems Key Design and Performance Aspects of Kalman-based Algorithms," $\mathrm{PhD}$ Thesis, Dept. of Engineering Sciences, Uppsala University, Sweden, 2011.

[7] G. Cili, H. Yanikomeroglu, and F. R. Yu, "CoMP adaptive channel estimation prediction filter design," Filed by Apple Inc., U.S. Patent Application No: $61 / 674,852$ (filing date: July 23, 2012).

[8] G. Cili, H. Yanikomeroglu, and F. R. Yu, "UE anchored down-selection for CoMP joint transmission cluster," Filed by Apple Inc., U.S. Patent Application No: 61/674,854 (filing date: July 24, 2012).

[9] 3GPP TR 36.814 V1.2.1, "Further advancements for EUTRA: Physical layer aspects," June 2009.

[10] International Telecommunication Union, "Guidelines for evaluation of radio interface technologies for IMT-Advanced, "Rep. ITU-R M.2135, 2008.

[11] D.S. Baum, J. Salo, G. Del Galdo, M. Milojevic, P. Kyösti, and J. Hansen, "An interim channel model for beyond-3G systems," in Proc. IEEE VTC'05, Stockholm, Sweden, May 2005.

[12] G. Cili, H. Yanikomeroglu, and F. R. Yu, "Cell switch off technique combined with coordinated multi-point (CoMP) transmission for energy efficiency in beyond-LTE cellular networks," in Proc. IEEE ICC'12 Workshop on Green Communications and Nenworking. Ottawa, ON, Canada, June 2012 .

[13] O. Amold, F. Richter, G. Fettweis, and O. Blume, "Power consumption modeling for different base station types in heterogeneous cellular networks," in Proc. Future Network and Mobile Summit 2010, pp. 1-8, June 2010

[14] G. Cili, H. Yanikomeroglu, and F. R. Yu, "Adaptive channel estimation for coordinated multipoint cellular communications," Filed by Apple Inc., U.S. Patent Application No: 61/710,267 (filing date: October 5, 2012). 


\section{THANK YOU !}

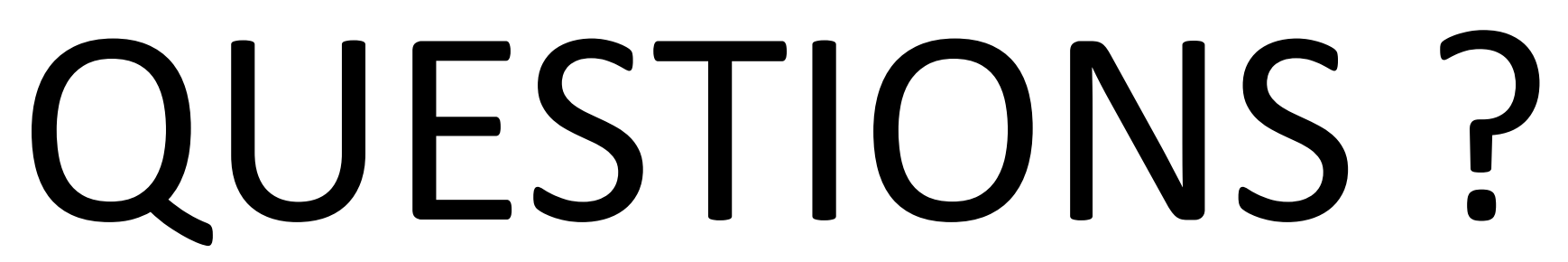

